\title{
Analysing the Impact of Traffic Incidents on the Travel Time Reliability of Freeway High-Occupancy Vehicle Lanes
}

\author{
Xianzhe Chen, ${ }^{1}$ Yajie Zou $\left(\mathbb{D},{ }^{2}\right.$ Jinjun Tang $\left(\mathbb{D},{ }^{3}\right.$ Yichuan Peng $\mathbb{D}^{2}$, \\ Lingtao $\mathrm{Wu}^{4}{ }^{4}$ and Yuming Jiang ${ }^{2}{ }^{2}$ \\ ${ }^{1}$ Department of Civil and Environmental Engineering, University of Washington, Seattle, WA 98195-2700, USA \\ ${ }^{2}$ Key Laboratory of Road and Traffic Engineering of Ministry of Education, Tongji University, Shanghai 201804, China \\ ${ }^{3}$ School of Traffic \& Transportation Engineering, Central South University, Changsha, Hunan 410075, China \\ ${ }^{4}$ Texas A\&M Transportation Institute, 3135 TAMU, College Station, Texas 77843-3135, USA
}

Correspondence should be addressed to Yichuan Peng; yichuanpeng1982@hotmail.com and Yuming Jiang; 14191@tongji.edu.cn

Received 23 August 2017; Accepted 30 April 2018; Published 5 August 2018

Academic Editor: Paolo Renna

Copyright (C) 2018 Xianzhe Chen et al. This is an open access article distributed under the Creative Commons Attribution License, which permits unrestricted use, distribution, and reproduction in any medium, provided the original work is properly cited.

\begin{abstract}
This study aims to examine the impact of traffic incidents occurring on general purpose lanes (GPLs) on the travel time reliability of high-occupancy vehicle (HOV) lanes on freeways and to evaluate the differences of travel time reliability on GPLs and HOV lanes under the same incident conditions. In this paper, an empirical travel time reliability analysis is conducted using the travel time and incident data collected between 2009 and 2012 on Interstate 5 and Interstate 405 of the Seattle metropolitan area. Three incident types (i.e., shoulder incident, single lane incident, and multiple lane incident) are considered. Two measures, percentilebased indicator and inflow-percentile travel time function, are used. The results suggest that incidents result in lower values of travel time reliability for all the measures. The results also show that multiple lane incident type has the most significant impact on the freeway route travel time reliability, while shoulder incident type has the least impact. Generally, HOV lanes have higher travel time reliability than GPLs under the same incident types. The findings in this study provide useful decision support for transportation agencies to improve travel time reliability on freeways.
\end{abstract}

\section{Introduction}

Travel time reliability is always considered as a key performance measure by transportation related departments and decision-makers since it is so critical for transportation system users. According to Chen et al. [1], reliability is commonly used in reference to the level of consistency in transportation service for a mode, trip, route, or corridor for a time period. Typically, reliability is viewed by travellers in relation to their experience. Therefore, travel time reliability is related to the quality of the transportation service provided, which explains why it is important to transportation system users. Especially, travel time reliability measures the extent of unexpected delay [2,3]. All the transportation system users are concerned with the travel time reliability. Personal users value travel time reliability because it can allow them to more wisely plan their trips and make better use of their time. On the other hand, the commercial companies require predictable and consistent travel time to keep themselves competitive.

The research plan for the reliability study of Future Strategic Highway Research Program suggested seven sources of travel time reliability [4-7]. They are traffic incidents, work zones, weather, fluctuations in demand, special events, traffic control devices, and inadequate base capacity. The traffic incidents are one of the most important sources affecting travel time reliability. Actually when incidents occur, one or two lanes are disrupted and the capacity of the roads is reduced, which causes traffic delay [8-10]. On the other hand, travellers may be informed about the incidents through different sources, such as Google map, and they may choose other routes. Therefore, more detailed analysis of travel time reliability under incident conditions should be conducted.

A high-occupancy vehicle (HOV) lane is a restricted traffic lane for vehicles with a driver and one or more passengers, including vanpools, carpools and transit buses [11-15]. 
The general purpose of HOV lanes is to encourage carpooling and reduce carbon emission by offering faster and more reliable trips than general purpose lanes (GPLs). So far, many studies have compared the characteristics of HOV lanes and GPLs. For instance, Burris and Lipnicky [16] use extensive data sets to compare user costs, specifically time and fuel costs, for two scenarios: a single reversible HOV lane and a GPL in each direction on the Katy Freeway in Houston, Texas-over a variety of mode and time shifts. Since the restrictions of $\mathrm{HOV}$ lanes and GPLs are different, travel time reliability on HOV lanes and GPLs are distinct as well. Nevertheless, few previous studies examined the travel time reliability on HOV lanes when the incidents occur on GPLs. In this circumstance, traffic incidents on GPLs can reduce the capacity of a freeway segment and generate a temporary bottleneck. As a result, drivers on GPLs may change to HOV lanes to avoid traffic congestion and the travel time reliability on HOV lanes can be affected. Therefore, it is necessary to investigate the travel time reliability on HOV lanes when the traffic incidents occur on GPLs.

The objective of this study is twofold, first to examine the impact of different kinds of traffic incidents occurring on freeway GPLs on the travel time reliability on HOV lanes and, second, to evaluate the differences of travel time reliability on GPLs and HOV lanes under same incident conditions. The findings in this work can provide useful decision support for transportation departments or traffic management agencies to improve travel time reliability on freeways. The major innovations of this study are as follows: first of all, the considered traffic incident dataset is highly comprehensive and includes detailed information (i.e., incident type, location, duration, affected lanes or shoulders, and other useful information) for each incident. Previous relevant studies often lack this kind of complete dataset. Secondly, the travel time reliability of HOV lanes and GPLs were evaluated and compared under normal situation and three incident types. Previous researchers have not fully examined the differences of travel time reliability between HOV lanes and GPLs under different incident situations. They mainly focused on examining the travel time reliability of GPLs under the normal situation and incident situation, which was not categorized by the severity. Last but not least, the difference of the travel time reliability of HOV lanes and GPLs under the various incidents is illustrated quantificationally.

\section{Literature Review}

There is some literature focused on the travel time reliability. Carrion and Levinson [18] comprehensively reviewed the existing research on travel time reliability and its value. They performed a meta-analysis to figure out the reasons behind the discrepancy among the reliability measures. However, they ignored the possible effects of study characteristics because of the disadvantages of meta-analysis. By using Bluetooth technology, Tang et al. [19] provided a framework for obtaining and analysing travel time data. They utilized the three statistical modelling alternatives to establish a relationship between travel times and known traffic parameters, which showed that it is possible to predict accurate mean travel times and travel time variability. The limitation is that the methods and techniques demonstrated in this research might be limited since the authors just used the data of Indiana. Li et al. [20] reviewed the modelling frameworks and empirical measurement paradigms which used to obtain willingness to pay for improved travel time reliability. In the context of Australian toll roads, the authors also estimated models to derive values of reliability, scheduling costs, and reliability ratios. Nevertheless, there was a data limitation as well because of the Australian data.

Some researchers focused on the influence of traffic accidents on freeway. Using a large set of traffic flow and accident data, Smith et al. [21] characterized urban freeway capacity reduction resulting from traffic accidents. The results showed that, for one or two lanes of three lanes blocked, the beta distribution provided a good representation of accident capacity reduction. However, the authors only considered three-lane segments in this study which was not realistic in reality. Knoop et al. [22] collected traffic counts from high-quality videos of the traffic flow around two accidents recorded from a helicopter. The results showed that the road capacity in the opposite direction was reduced by half and the capacity of the road in the direction of the accident was reduced by more than half because not all lanes were in use. However, there were only two accidents considered in the analysis. Based on a large dataset of registered traffic accident data and empirical traffic flow data, Tu et al. [23] conducted an empirical travel time reliability analysis. The authors found that the travel time accidents resulted in both higher travel time variability and higher probabilities of traffic breakdown on freeways. Nevertheless, the severity of traffic accident and the combination of other factors like weather conditions and road geometry were not considered.

Some studies have focused on the travel time reliability on HOV lane, but very few of them examined the impact of traffic incidents on travel time reliability of HOV lanes. Turner et al. [24] examined the travel time reliability of three HOV lanes in Texas: Katy (I-10) Freeway, Houston, East R.L. Thornton (I-30) Freeway, Dallas, and North (I45) Freeway, Houston. They compared travel time reliability between the HOV lanes and the freeway mainlines and utilized statistical tests to evaluate the differences in travel time reliability. However, the travel time data might be not reliable since they were collected using the computerized license matching technique and cellular phone travel time reports. To improve the accuracy of speed on HOV lanes, Loudon [25] used data from heavily congested California freeways to obtain a mathematical model to estimate the vehicle speed on $\mathrm{HOV}$ lane, on the basis of the $\mathrm{V} / \mathrm{C}$ ratio in the $\mathrm{HOV}$ lane and the $\mathrm{V} / \mathrm{C}$ ratio in the adjacent lanes. Nevertheless, the research is based on the analysis of only a single freeway with a buffer-separated, limited entry HOV lane. Further research based on the HOV lanes of different types should be conducted. Ye et al. [26] examined travel time savings of the barrier-separated high-occupancy vehicle lane during incident conditions in California. They compared travel time savings during incident conditions to nonincident conditions for the range of incidents in the incident matrix. It is good to consider the travel time savings of HOV lanes 
under incident conditions, but their study did not take the detailed incident type into consideration, such as single lane incident.

Up to date, although some studies have investigated the freeway travel time reliability, the impacts of traffic incidents on freeway operation, and travel times of HOV lanes, very few studies have comprehensively evaluated freeway travel time reliability of HOV lanes, when different incidents occur on GPLs. Thus, there is a need to further examine the effect of incidents on travel time reliability of HOV lanes using datasets with complete information (e.g., the incident type, location, duration, affected lanes or shoulders, and other useful information). The primary objective of this study is to investigate the relationship between traffic incidents and travel time reliability on GPL and HOV lanes of freeway routes. To accomplish the objective, different travel time measures were calculated using four years of travel time and incident data collected on Interstate 5 and Interstate 405 in the Seattle area.

\section{Methodology}

According to Lomax and Margiotta [27], reliability measures in transportation service can be grouped into three main categories: statistical range methods, buffer time methods, and tardy-trip measures. In this study, two improved percentilebased measures are adopted in this study. They are percentilebased indicator and inflow-percentile travel time function, which are briefly introduced below.

3.1. Percentile-Based Indicator. The percentile-based measures are widely used by researchers to evaluate travel time reliability, which usually appear as "variability" measures. According to Tu et al. [28], one of the benefits of choosing the percentile-based indicators was that their statistical properties are more robust than standard deviation, mean, or other moment-based indicators. Besides, the measures allow comparisons of reliability over different route distances. This paper adopted a statistical range method proposed by Tu et al. [29] to quantify travel time reliability. Through the percentilebased measure, travel time reliability in each traffic condition on HOV lanes and GPLs is obtained. There are four traffic conditions considered in this study in total: normal condition with no incidents, incidents on the shoulder, incidents on a single lane, and incidents on multiple lanes. Travel time variability is reflected by the difference between the 90th and 10th percentile travel time in traffic condition $\mathrm{k}$ through (1). The larger the travel time variability is, the worse the travel time reliability is.

$$
T T V_{k}=T T 90_{k}-T T 10_{k}
$$

where

$T T V_{k}=$ the travel time reliability (seconds/mile) in traffic condition $\mathrm{k}$;

TT $90_{k}=$ the 90th percentile travel time per unit road distance (seconds/mile) in traffic condition $\mathrm{k}$;

$T T 10_{k}=$ the 10 th percentile travel time per unit road distance (seconds/mile) in traffic condition $\mathrm{k}$; $\mathrm{k}=$ normal condition with no incidents, incidents on the shoulder, incidents on a single lane, and incidents on multiple lanes.

3.2. Inflow-Percentile Travel Time Functions. According to $\mathrm{Tu}$ et al. [29], the inflow-travel time continuous function is defined as follows:

$$
\begin{aligned}
& \widehat{T T} X X \\
& = \begin{cases}f_{1}(q)=t_{f}^{X X} \cdot\left(1+\alpha_{1} \cdot\left(\frac{q}{\lambda_{1}}\right)^{\beta_{1}}\right) & q \leq \lambda_{2} \\
f_{2}(q)=t_{f}^{X X} \cdot\left(\alpha_{2} \cdot\left(\frac{q-\lambda_{2}}{\lambda_{2}}\right)+\gamma_{1}\right) & q \geq \lambda_{2}\end{cases}
\end{aligned}
$$

where $\widehat{T T} X X$ is the percentile travel time (e.g., TT10, TT50, and TT90); $\lambda_{1}$ is the critical transition flow; $\lambda_{2}$ is the critical capacity flow; $\alpha_{1}, \alpha_{2}, \gamma_{1}$, and $\beta_{1}$ are parameters. All parameters in (2) can be determined by fitting the estimated travel time $\widehat{T T}$ to the observed travel time $T T$ and minimizing the squared errors. Since (2) is a continuous function, parameter $\gamma_{1}$ can be calculated as $\gamma_{1}=1+\alpha_{1} \cdot\left(\lambda_{2} / \lambda_{1}\right)^{\beta_{1}}$. As discussed by $\mathrm{Tu}$ et al. [29], the proposed inflow-travel time continuous function can be useful for traffic management measures (e.g., ramp metering, variable speed limits) and has been considered in some previous travel time reliability studies.

\section{Data Description}

The study area was located in the Seattle metropolitan area, which is the largest urban area in Washington State. Travel time reliability of Interstate 5 and Interstate 405 was examined. There were three routes in total. They are Interstate 405 North, Interstate 405 South, and Interstate 5 South. Interstate 5 North is excluded from the analysis due to the incomplete loop detector data on the HOV lane. Figure 1 shows the locations of Interstate 5 and Interstate 405 . Interstate 5 , or $\mathrm{I}-5$, is the main interstate highway on the West Coast of the United States, running from the Canadian to Mexican borders of the United States [30]. Interstate 405, or I-405, is a 30-mile auxiliary route of I-5 that bypasses Seattle east of Lake Washington. For this research, the southbound I-5 study section is 15.28 miles long, the northbound I-405 study section is 7.16 miles long, and the southbound I-405 section is 6.88 miles long.

These locations were chosen in order to hold the speed limits and the number of lanes constant along each route. All routes maintain a speed limit of $60 \mathrm{mph}$, and the I-5 route maintains three GPLs along its length while the I- 405 route has two GPLs. All routes also have one continuous HOV lane operating on the left side. Other geometrical characteristics, such as lane and shoulder widths, are reasonably consistent. It should also be mentioned that the HOV lanes are consistently accessible along the study site.

The travel times used in this study were calculated using traffic flow data measured by single loop detectors between 2009 and 2012 [17]. The I-5 study section includes 33 detector locations, and the I-405 study section contains 


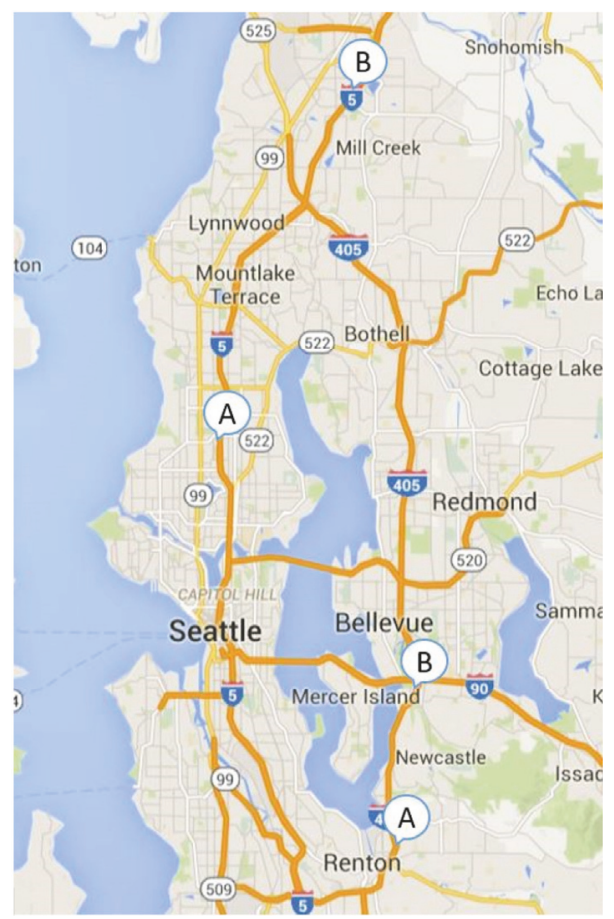

FIgURE 1: Locations of I-5 (Top) and I-405 (Bottom) [17].

15. These detectors are embedded under the roadway with an average spacing of about 0.5 miles and report aggregate data at 20-second intervals. For this study, the 20 -second data was further aggregated into five-minute chunks. For the calculation of travel time percentiles and variability, only the time periods between 5:00 AM and 7:30 PM were considered. This is because most trips happen during daytime, which is more typical for data analysis. Each loop detector data point contains the detector's milepost, a time stamp, and the 5minute volume and occupancy measured by the detector. These four parameters are then used to calculate the route travel time. The detailed calculation of the route level travel times is explained in a previous study [17].

There were only minor changes to the infrastructure along these routes during the study time period from 2009 to 2012. No additional lanes were added on these routes, and the annual average daily traffic of the roadways changed slightly. Therefore, there is no need to study the travel time reliability under different traffic demand, since the demand of the study site is consistent through four years. Only minor improvements were made to improve the travel information available to drivers, such as the installation of Intelligent Transportation System (ITS) equipment, which could have enhanced reliability on the route.

Incident data from the Washington Incident Tracking System (WITS), a database containing highly detailed information for each incident, was collected from the beginning of 2009 to the end of 2012, which matched the time span of the travel time data. The Washington State Incident Response Team collected detailed information for every incident, including the incident type, location, duration, affected lanes or shoulders, and other useful information. Three incident types, i.e., shoulder incident, single lane incident, and multiple lane incident, which occur on GPLs, are considered in our analysis. Once incidents occur on the HOV lane, the HOV lane would be totally blocked since there is only one HOV lane in each route. Thus, the HOV incident type is not considered. However, obviously incidents on HOV lanes have the most serious impacts on the travel time reliability of $\mathrm{HOV}$ lanes.

\section{Results and Discussion}

Travel time reliability of three routes, i.e., I-5 South, I-405 North, and I-405 South, was calculated and analysed under four conditions. The four conditions are normal condition with no incidents, incidents on the shoulder, incidents on a single lane, and incidents on multiple lanes.

5.1. Percentile-Based Indicator. Figures 2, 3, 4, and 5 show the comparison of percentile travel time and travel time variability between HOV lanes and GPL of I-5 South, I-405 North, and I-405 South.

5.1.1. Impact of Shoulder Incidents. TT10 of HOV lanes and GPLs and TT50 of HOV lanes and GPLs in each route are almost the same under shoulder incident, while the difference of TT90 between HOV lanes and GPLs is large, with the results of $9.98 \%$ in I-5 South, $40.81 \%$ in I-405 North, and $22.74 \%$ in I-405 South. TT10 and TT50 represent the low traffic volume conditions. TT90 are the travel time values observed in the peak hours, and the freeway bottleneck can be easily caused by shoulder incidents. Therefore, the differences of TT90 between HOV lanes and GPLs are 


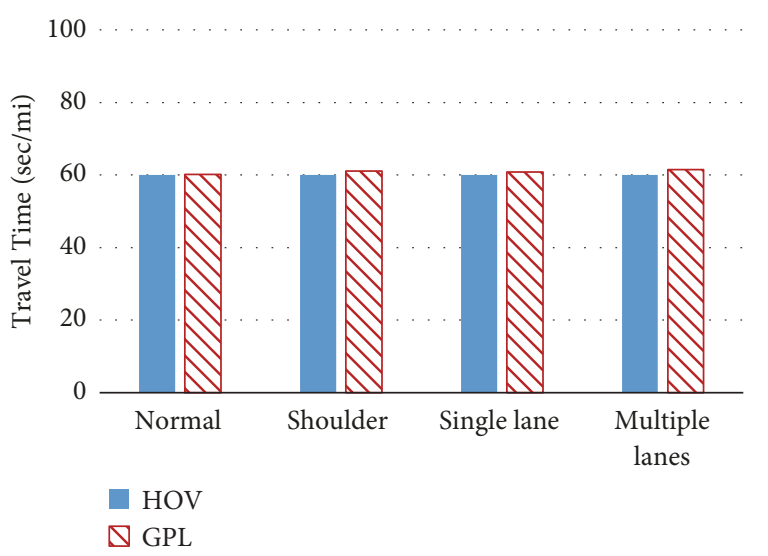

(a) TT10 in I-5 South

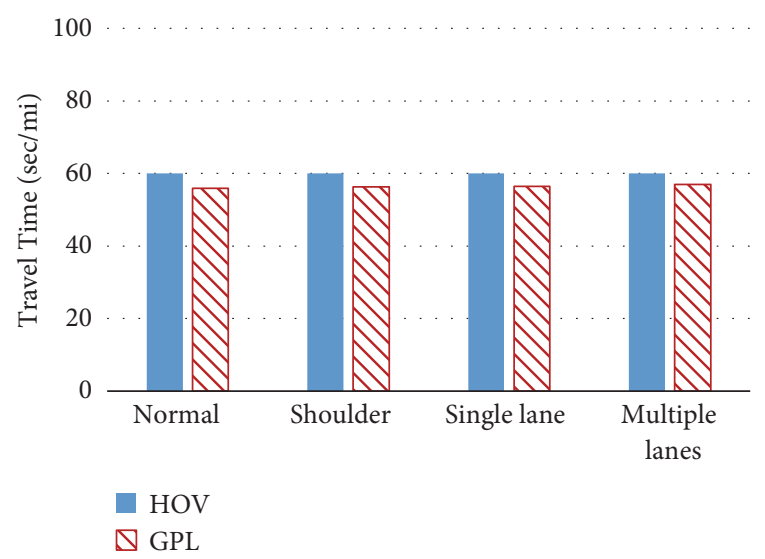

(b) TT10 in I-405 North

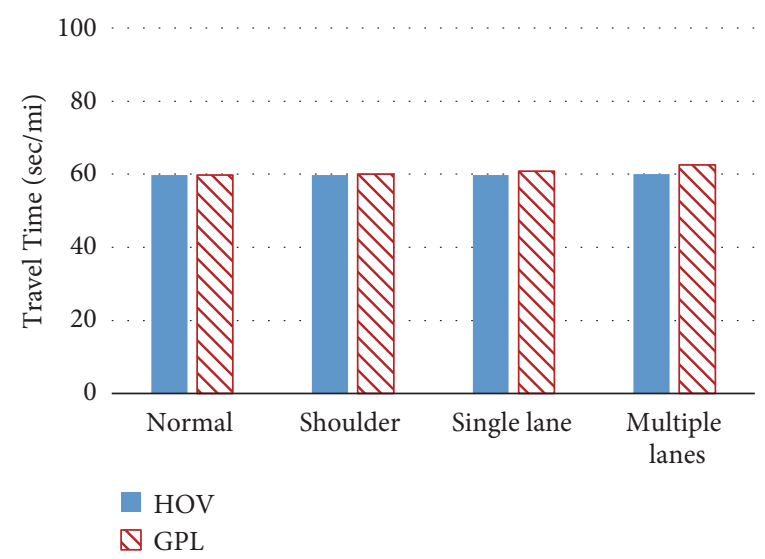

(c) TT10 in I-405 South

FIgURE 2: Comparison of 10th percentile travel time between HOV lanes and GPLs on I-5 South, I-405 North, and I-405 South. (a) TT10 in I-5 South. (b) TT10 in I-405 North. (c) TT10 in I-405 South.

remarkable although shoulder incidents are minor incidents. Once the GPLs become congested due to incidents, the single-occupant vehicles are not able to be driven on HOV lanes. Thus, the traffic incident that occurred on GPLs generally has a slight impact on HOV lanes. On the other hand, if the incidents happen on HOV lanes, the drivers who originally drive on HOV lanes can shift to GPLs to avoid worse traffic jams.

The differences of HOV lanes and GPLs in TTV under shoulder incident condition are $29.37 \%$ in I-5 South, $85.36 \%$ in I-405 North, and $48.54 \%$ in I-405 South based on GPLs. Thus HOV lanes have a better travel time reliability than GPLs since TTV accounts for travel time reliability.

5.1.2. Impact of Single Lane Incidents. TT10 in HOV lanes and GPLs are almost the same in all the three routes under the single lane incident condition. However, the travel time differences between HOV lanes and GPLs can be observed for TT50 and TT90, which is different from the shoulder incident. The differences in TT50 are $2.68 \%$ in I-5 South, $13.47 \%$ in I- 405 North, and $20.02 \%$ in I-405 South, while the differences in TT90 are $12.72 \%$ in I-5 South, $42.96 \%$ in I-405 North, and $4.75 \%$ in I-405 South. Since single lane incidents can block one of the travel lanes on freeway road segments and induce a bottleneck at a relatively low traffic flow rate, this incident type can have a more obvious impact on the travel time than the shoulder incident.

The differences of TTV between HOV lanes and GPLs in single lane incidents are $32.74 \%$ in I-5 South, $85.91 \%$ in I-405 North, and $7.66 \%$ in I- 405 South. Under the impact of single lane incidents, HOV lanes can provide a better travel time reliability than GPLs.

5.1.3. Impact of Multiple Lane Incidents. The difference of TT10 between HOV lanes and GPLs is minor under the impact of multiple lane incidents, while the differences are noticeable for TT50 and TT90. The differences for TT50 are $11.74 \%$ in I-5 South, $23.24 \%$ in I-405 North, and 20.61\% in I-405 South, while the differences for TT90 are $26.88 \%$ in I-5 South, $26.86 \%$ in I-405 North, and $34.48 \%$ in I-405 South. The differences are more significant than those in single lane incidents. Multiple lane incidents are the most serious incident type among all the three incident types, which can block multiple lanes. Therefore, the threshold traffic flow value for traffic breakdown is smaller for multiple lane incidents than the single lane incident and shoulder 


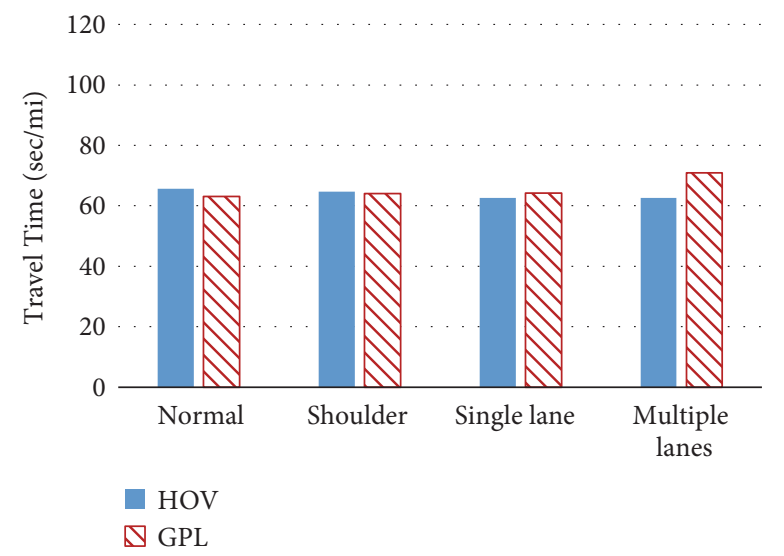

(a) TT50 in I-5 South

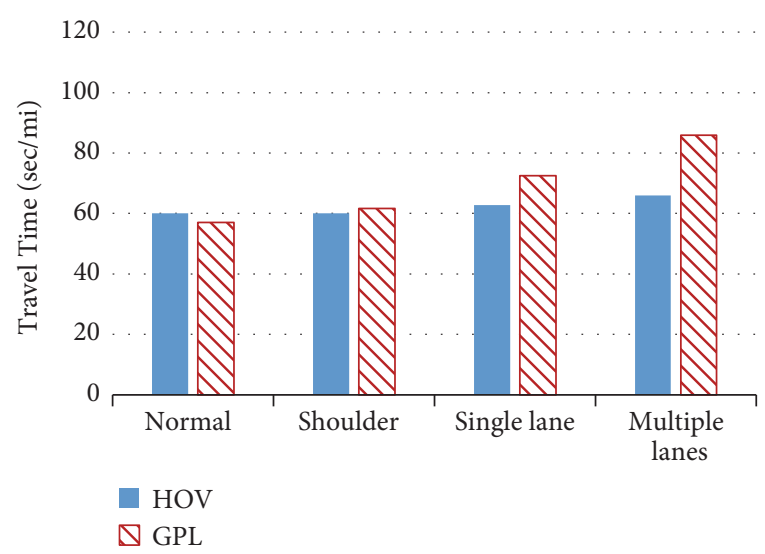

(b) TT50 in I-405 North

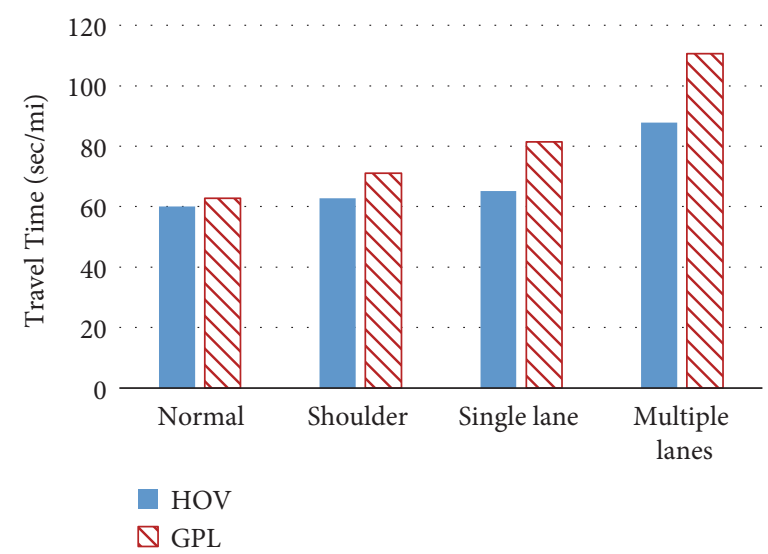

(c) TT50 in I-405 South

FIgure 3: Comparison of 50th percentile travel time between HOV lanes and GPLs in I-5 South, I-405 North, and I-405 South. (a) TT50 in I-5 South. (b) TT50 in I-405 North. (c) TT50 in I-405 South.

incident. It is probably because HOV lanes and GPLs are both influenced heavily by the multiple lane incidents and the situation becomes worse with the increase in traffic volumes. For multiple lane incidents, travel times on HOV lanes are shorter than those on GPLs, since the single-occupant vehicles are not allowed to use the HOV lanes.

Under multiple lane incident circumstance, the differences between HOV lanes and GPLs in TTV are $55.36 \%$ in I-5 South, $41.81 \%$ in I-405 North, and $46.90 \%$ in I-405 South based on GPLs, meaning that HOV lanes have better travel time reliability.

5.1.4. Comparison of Impacts of Different Incidents. All the incident types have negative influence on travel time reliability. Nevertheless, the level of impact is different. To examine the travel time reliability of HOV lanes under different incidents, the difference of travel time reliability between the incident situations and normal situations is calculated. The difference of travel time reliability between shoulder incidents and normal situation is $0.00 \%$ in I-5 South, $31.64 \%$ in I-405 North, and $2.67 \%$ in I-405 South based on the normal situation. The difference of travel time reliability between single lane incidents and normal situation is $25.99 \%$ in I-5 South, 36.88\% in I-405 North, and $126.20 \%$ in I-405 South based on the normal situation. The difference of travel time reliability between multiple lane incidents and normal situation is $28.17 \%$ in I-5 South, $970.29 \%$ in I-405 North, and $213.76 \%$ in I-405 South based on the normal situation. The travel time reliability of GPLs under different incidents follows the similar pattern as well. Therefore, among all the incident types, shoulder incident condition results in the best travel time reliability, single lane incident condition ranks second, and multiple lane incident condition causes the worst travel time reliability. As expected, more serious incidents generally increase the uncertainty of travel time.

The difference of TTV between HOV lanes and GPLs in each route also varies under different incident types. For example, in I-5 South, it is $-0.87 \%$ under normal condition, $29.37 \%$ in shoulder incidents, $32.74 \%$ in single lane incidents, and $55.36 \%$ in multiple lane incident situations. Thus when incidents occurred on GPLs, the traffic on HOV lanes will not be significantly affected and HOV lanes generally provide a much better travel time reliability.

5.1.5. Comparison of I-5 and I-405. The data of I- 405 South and I-405 North were taken as an average to compare with 


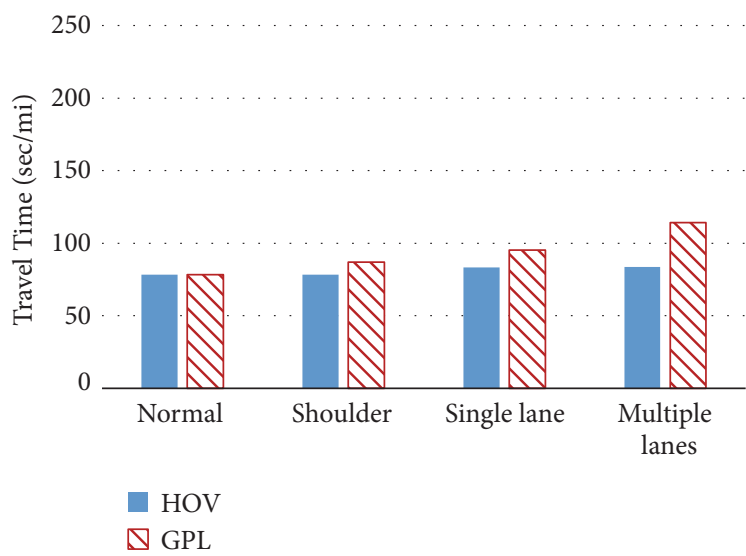

(a) TT90 in I-5 South

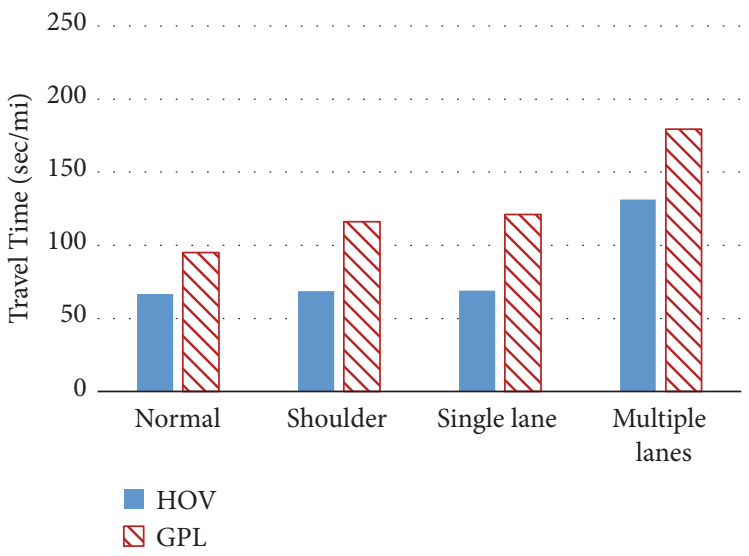

(b) TT90 in I-405 North

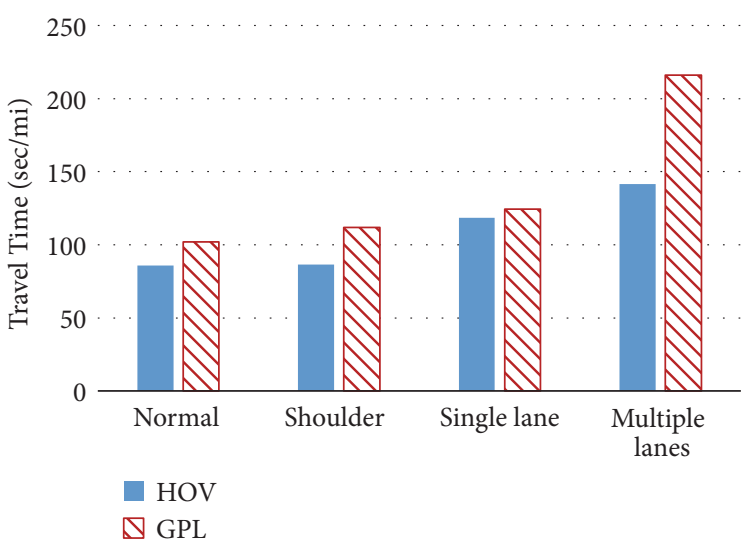

(c) TT90 in I-405 South

FIgURE 4: Comparison of 90th percentile travel time between HOV lanes and GPLs on I-5 South, I-405 North, and I-405 South. (a) TT90 in I-5 South. (b) TT90 in I-405 North. (c) TT90 in I-405 South.

that of I-5 South. It is obvious that I-5 has a better travel time reliability than I-405 for GPL since the travel time variability indexes of I-405 are larger than those of I-5 overall. However, on HOV lanes, I-5 has a higher reliability under single lane incidents and multiple lane incidents, while travel time reliabilities of I-5 and I-405 on average are quite the same under the normal and shoulder incident conditions. For GPLs, one possible explanation is that I-5 has three general purpose lanes while I-405 only has two. Therefore, I-5 is more reliable than I-405 in normal situation because vehicles in I-5 have more lane options to shorten their travel time. On the other hand, once the incidents happen, vehicles in I-5 have a higher flexibility to choose lanes to avoid the queue. In terms of HOV lanes, the travel time reliabilities under normal condition and shoulder incidents, the most slight incident type, are quite the same since both I-5 and I-405 have only one HOV lane. Nevertheless, when more serious incident types occur, the advantage of more GPLs in I-5 is prominent, resulting in better travel time reliability. To sum up, the number of GPLs does influence travel time reliability. More general purpose lanes can result in higher travel time reliability on GPLs when traffic incidents occur.
Another noticeable difference between I-5 and I-405 is that I-5 is smooth, while I-405 has distinguishable grade difference in the Washington State, which can also influence the travel time reliability. The grade difference can have indirect impacts on driving behaviour, resulting in worse travel time reliability. Therefore, travel time reliability of I-5 is generally better.

5.2. Inflow-Percentile Travel Time Function. In order to draw the inflow-percentile travel time function, the vehicle inflow was categorized, as shown in Table 1 . The vehicle inflow between $0 \mathrm{veh} / \mathrm{h} / \mathrm{ln}$ and $100 \mathrm{veh} / \mathrm{h} / \mathrm{ln}$ is marked as 1 , and the vehicle inflow between $100 \mathrm{veh} / \mathrm{h} / \mathrm{ln}$ and $200 \mathrm{veh} / \mathrm{h} / \mathrm{ln}$ is marked as 2 . The rest can be done in the same manner.

The data sample sizes were also examined. The inflow categories with too few sample size were not considered. Therefore category 1 was not considered in the study. In this study, the sample sizes in single lane incident and multiple lane incidents are too small, and the data of I-5 is not complete. Therefore, only the data with sufficient sample sizes of I-405 South and I-405 North under normal situations and shoulder lane incidents are considered. The horizontal axis 
TABLE 1: Table of inflow-percentile travel time of GPL I-405 South under normal situation.

\begin{tabular}{|c|c|c|c|c|c|}
\hline & TT10 (sec/mi) & TT50 (sec/mi) & TT90 (sec/mi) & Sample Size & Inflow $(v e h / h / l n)$ \\
\hline 1 & 59.74 & 60.00 & 160.20 & 153 & $0-100$ \\
\hline 2 & 59.74 & 60.00 & 60.00 & 2060 & $100-200$ \\
\hline 3 & 59.74 & 60.00 & 60.00 & 5127 & $200-300$ \\
\hline 4 & 59.74 & 60.00 & 60.00 & 5329 & $300-400$ \\
\hline 5 & 59.74 & 60.00 & 60.00 & 5677 & $400-500$ \\
\hline 6 & 59.74 & 60.00 & 62.57 & 5879 & $500-600$ \\
\hline 7 & 59.74 & 60.00 & 111.83 & 5693 & $600-700$ \\
\hline 8 & 59.74 & 60.00 & 119.35 & 8546 & $700-800$ \\
\hline 9 & 59.74 & 61.15 & 117.39 & 16235 & $800-900$ \\
\hline 10 & 59.74 & 63.87 & 111.87 & 24496 & $900-1000$ \\
\hline 11 & 60.00 & 63.44 & 105.27 & 35361 & $1000-1100$ \\
\hline 12 & 60.00 & 63.36 & 98.85 & 43503 & $1100-1200$ \\
\hline 13 & 60.00 & 63.51 & 93.39 & 34495 & $1200-1300$ \\
\hline 14 & 60.10 & 64.23 & 90.54 & 26277 & $1300-1400$ \\
\hline 15 & 60.29 & 65.76 & 89.87 & 17840 & $1400-1500$ \\
\hline 16 & 60.55 & 68.53 & 90.98 & 6884 & $1500-1600$ \\
\hline 17 & 60.86 & 69.73 & 90.63 & 2134 & $1600-1700$ \\
\hline 18 & 60.75 & 69.63 & 95.15 & 455 & $1700-1800$ \\
\hline 19 & 59.74 & 66.57 & 104.43 & 139 & $1800-1900$ \\
\hline 20 & 59.74 & 65.41 & 96.61 & 126 & $1900-2000$ \\
\hline 21 & 59.92 & 65.69 & 94.41 & 137 & $2000-2100$ \\
\hline 22 & 60.16 & 65.94 & 102.96 & 102 & $2100-2200$ \\
\hline
\end{tabular}

range and vertical axis range of the figures were adjusted so that they can be compared clearly. The results are shown in Figures 6 and 7.

In the inflow-percentile travel time function, the line of 90th percentile travel time is thought to be important, since it presents nearly the worst travel time. Besides, the ranges of 10th percentile travel time and 50th percentile travel time are relatively small, while the ranges of 90th percentile travel time are obvious and large, which is useful for the researchers to study the travel time reliability. The inflow where the vehicle travel time begins to change in the line of 90th percentile travel time is called critical transition flow [29].

For I-405 South GPL, the critical transition flow in shoulder incident figure happens first with the increase of the inflow, which is expected, because the incidents will reduce the freeway segment capacity, triggering the congestions happening earlier than the normal situation. In addition, the peak point and the fluctuations of the 90th percentile travel time line is larger for the shoulder incident, meaning that the worst travel time in the shoulder incident is larger, and the travel time is more unstable.

For I-405 South HOV lanes, it can be observed that the overall line shapes of normal situation and shoulder incidents are very alike, meaning that shoulder incidents which happen on GPLs barely have significant impacts on the HOV lanes. This is mainly because most vehicles travelling on GPLs cannot transfer into HOV lanes since there is only one person in vehicle. Therefore the travel time in HOV lanes will not be effectively affected.

Under the normal situation, I-405 South GPL and I-405 South HOV lanes were compared. The critical transition flow in GPL is $600 \mathrm{veh} / \mathrm{h} / \mathrm{ln}$, while it is approximately $800 \mathrm{veh} / \mathrm{h} / \mathrm{ln}$ for the HOV lanes, meaning that the travel time on GPL is affected first with the increase of the inflow. Besides, the peak point and the fluctuations of the 90th percentile travel time line are larger in the GPL, meaning that the worst travel time in the GPL is larger and the travel time is more unstable. Therefore, obviously HOV lanes are more reliable than GPLs under the normal situation. I-405 South GPL and I-405 South HOV lanes under the shoulder incident situation are pretty like the normal situation: the critical transition flow in GPL happens earlier, and the peak point and the fluctuations of the 90th percentile travel time line in GPL are larger.

Inflow-percentile travel time function of I-405 North HOV and I-405 North GPL under normal situation and shoulder incident is shown in Figure 7. The sample sizes of I-405 GPL and HOV North under the shoulder incident situation are relatively small, which explains why the shapes of the lines are somewhat weird. However, it can still be seen that the I-405 North follows the same pattern as I-405 South.

\section{Conclusions}

This study aims to understand the impacts of traffic incidents occurring on the GPLs on the travel time reliability of $\mathrm{HOV}$ 


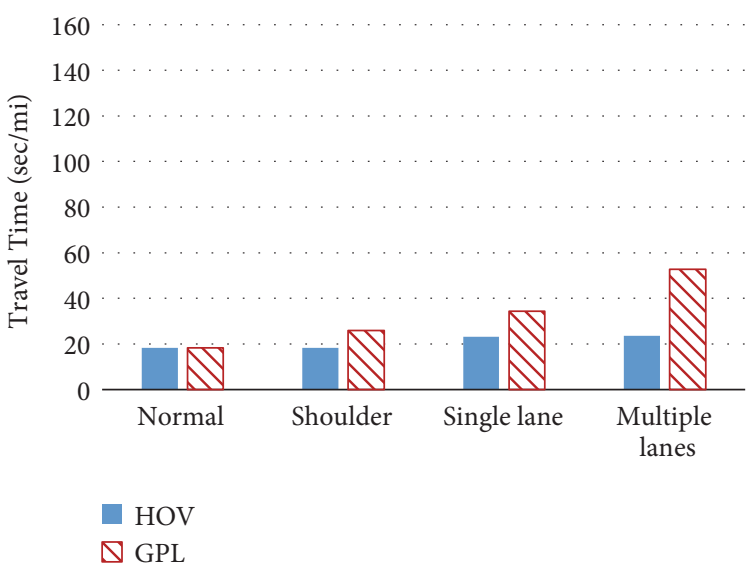

(a) TTV in I-5 South

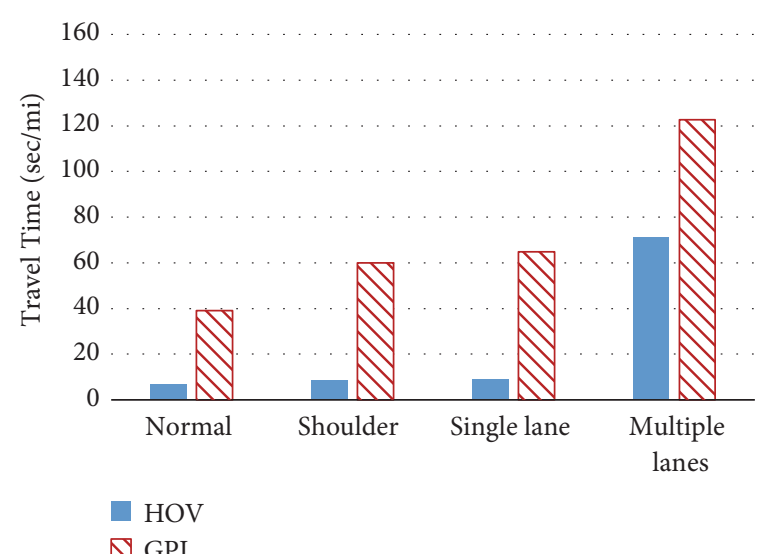

(b) TTV in I-405 North

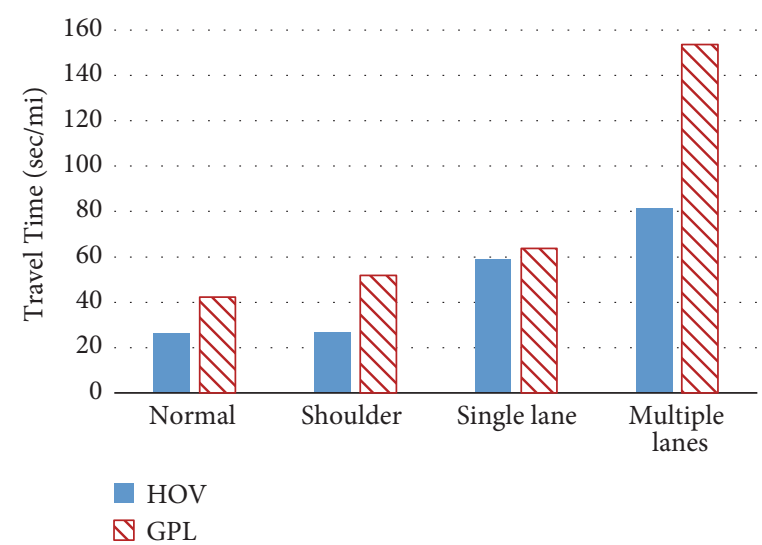

(c) TTV in I-405 South

FIgURE 5: Comparison of travel time variability between HOV lanes and GPLs on I-5 South, I-405 North, and I-405 South. (a) TTV in I-5 South. (b) TTV in I-405 North. (c) TTV in I-405 South.

lanes and to evaluate the differences of travel time reliability on GPLs and HOV lanes under the same incident conditions. Three incident types were considered: shoulder incidents, single lane incidents, and multiple lane incidents. The travel time and incident data were collected between 2009 and 2012 on Interstate 5 and Interstate 405 of the Seattle metropolitan area.

To evaluated travel time reliability, two measures were utilized. They are percentile-based indicator and inflow-percentile travel time function. For the percentilebased indicator, first of all, impacts of shoulder incidents were examined. The result shows that TT10 on HOV lanes and GPLs in each route are quite the same in shoulder incidents as well as TT50, while TT90 on GPLs are more than those on HOV lanes. Then, impacts of single lane incidents were discussed. TT10 on HOV lanes and GPLs in three routes are nearly the same, while TT50 and TT90 on GPLs are more than those on HOV lanes in single lane incidents. Next, influences of multiple lane incidents were also examined. Similar to single lane incidents, TT10 on
HOV lanes and GPLs in each route under multiple lane incidents are close, while TT50 and TT90 for GPLs are more than those for $\mathrm{HOV}$ lanes. Through all the comparisons among three incident types, HOV have higher travel time reliability than GPLs under the same incident types. The impacts of three incident types were also examined. It shows that all the incident types have negative influence on travel time reliability. Multiple lane incident type influences freeway route travel time reliability the most, while shoulder incident type affects travel time reliability the least. The more serious the incidents are, the less travel time reliability is.

In terms of inflow-percentile travel time function, only the data of I-405 South and I-405 North under normal situation and shoulder lane incidents are utilized because of the limited sample size. The results show that, in I-405 South GPL, the shoulder incident situation is more likely to have congestion than the normal situation under the same inflow level. In addition, the worst travel time in the shoulder incident is larger, and the travel time is more unstable. For I-405 South HOV lanes, it illustrates that shoulder incidents, 


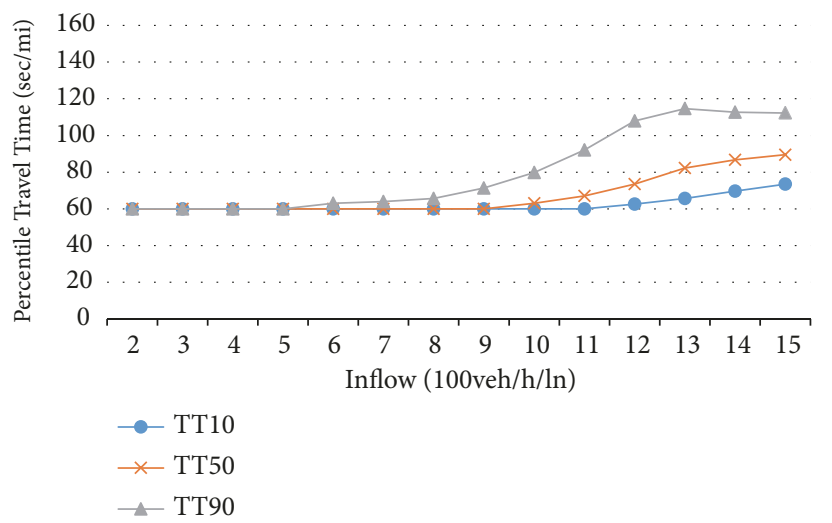

(a) Inflow-Percentile Travel Time in I-405 South HOV (normal situation)

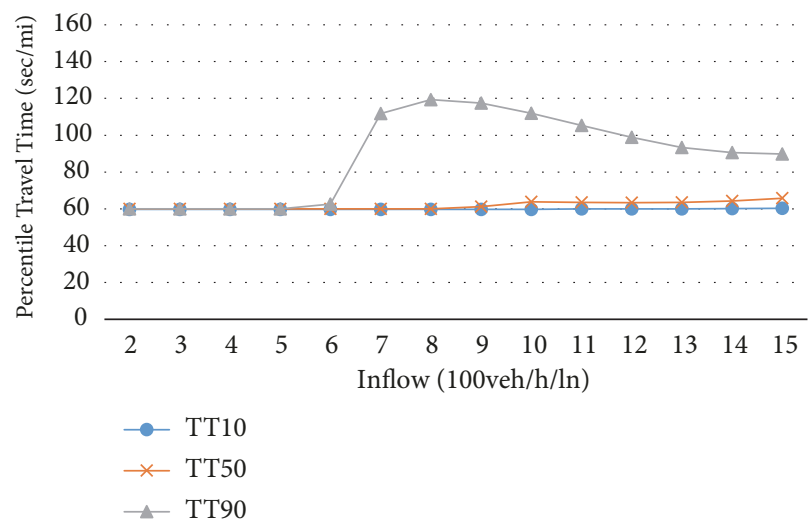

(c) Inflow-Percentile Travel Time in I-405 South GPL (normal situation)

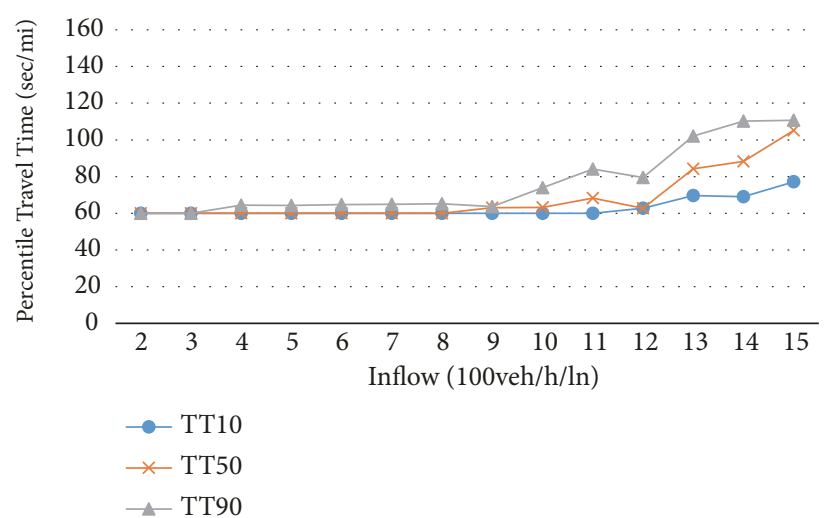

(b) Inflow-Percentile Travel Time in I-405 South HOV (shoulder incident)

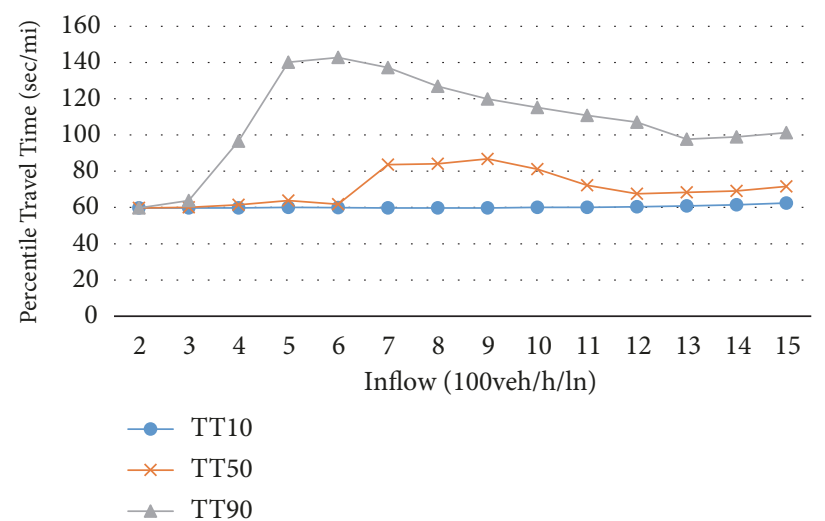

(d) Inflow-Percentile Travel Time in I-405 South GPL (shoulder incident)

FIGURE 6: Inflow-Percentile travel time function in I-405 South under normal situation and shoulder incident. (a) I-405 South HOV (normal situation). (b) I-405 South HOV (shoulder incident). (c) I-405 South GPL (normal situation). (d) I-405 South GPL (shoulder incident).

which happen on GPLs, barely have significant impacts on the HOV lanes. Under both the normal situation and shoulder incident situation, I-405 South GPL is more possible to have congestions than I-405 South HOV. Besides, the worst travel time in the GPL is larger, and the travel time is more unstable, meaning that HOV lanes are more reliable than GPLs. The figures of I-405 North follow the same pattern as those of I405 South.

Among different incident types, transportation agencies are suggested to take priority to handle multiple lane incidents because this incident type can cause the worst travel time reliability. The findings in this study can provide useful decision support for transportation agencies to improve travel time reliability on freeways $[31,32]$.

There are also some limitations for this research. For example, this study only evaluates the travel time reliability performances on three routes on I-405 and I-5. Multiple routes in other regions should be examined to further validate the findings from this study. Future research can include but is not limited to examining the incidents impacts on twofreeway segments with the same lane numbers, while one has
HOV lanes and one does not have HOV lanes. However, the other factors of the two-freeway segments should be almost the same, such as weather, roadway geometry, and traffic flow.

\section{Disclosure}

This research was presented in the 95th Transportation Research Board Annual Meeting.

\section{Conflicts of Interest}

There are no conflicts of interest.

\section{Acknowledgments}

This research is sponsored jointly by the National Natural Science Foundation of China (Grant nos. 51608386 and 71601143), Shanghai Pujiang Program (16PJC088), and Shanghai Sailing Program (Grant no. 16YF1411900). 


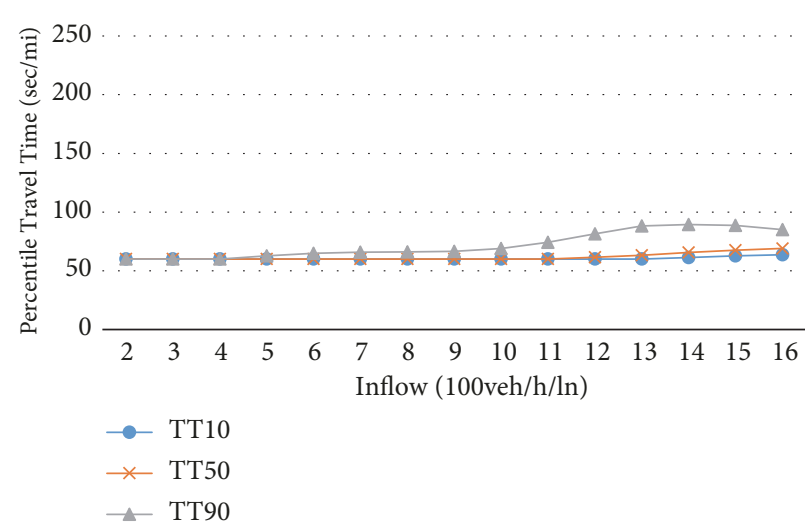

(a) Inflow-Percentile Travel Time in I-405 North HOV (normal situation)

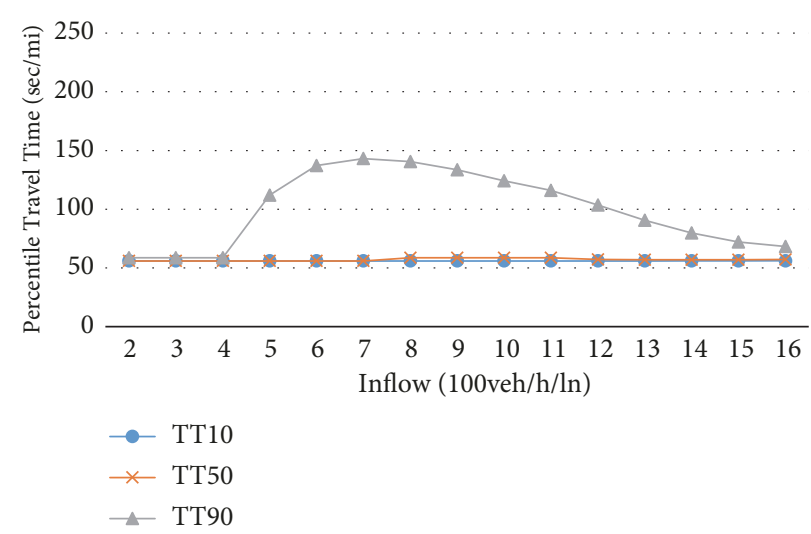

(c) Inflow-Percentile Travel Time in I-405 North GPL (normal situation)

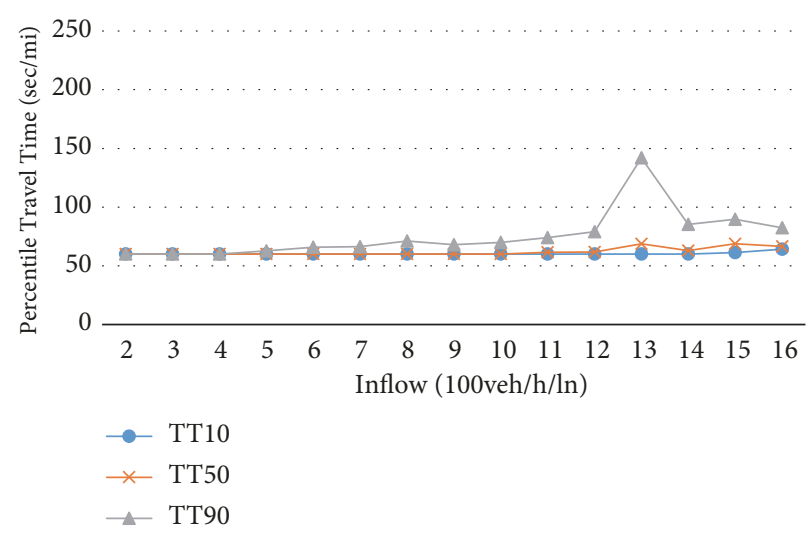

(b) Inflow-Percentile Travel Time in I-405 North HOV (shoulder incident)

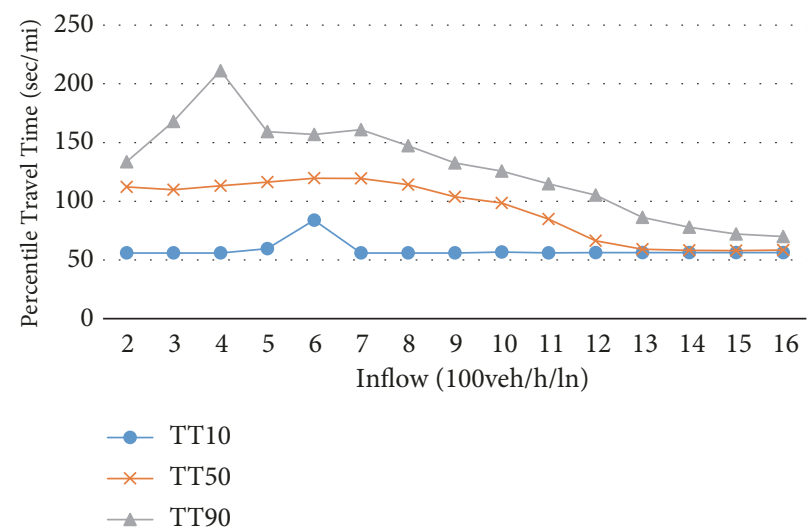

(d) Inflow-Percentile Travel Time in I-405 North GPL (shoulder incident)

FiguRE 7: Inflow-Percentile travel time function in I-405 North under normal situation and shoulder incident. (a) I-405 North HOV (normal situation). (b) I-405 North HOV (shoulder incident). (c) I-405 North GPL (normal situation). (d) I-405 North GPL (shoulder incident).

\section{References}

[1] P. Chen, R. Tong, G. Lu, and Y. Wang, “The $\alpha$-reliable path problem in stochastic road networks with link correlations: A moment-matching-based path finding algorithm," Expert Systems with Applications, vol. 110, pp. 20-32, 2018.

[2] K. Lyman and R. L. Bertini, "Using travel time reliability measures to improve regional transportation planning and operations," Transportation Research Record, no. 2046, pp. 1-10, 2008.

[3] J. Tang, F. Liu, Y. Wang, and H. Wang, "Uncovering urban human mobility from large scale taxi GPS data," Physica A: Statistical Mechanics and its Applications, vol. 438, pp. 140-153, 2015.

[4] W. Pu, "Analytic relationships between travel time reliability measures," Transportation Research Record, vol. 2254, pp. 122130, 2011.

[5] Y. Yan, S. Zhang, J. Tang, and X. Wang, "Understanding characteristics in multivariate traffic flow time series from complex network structure," Physica A: Statistical Mechanics and Its Applications, vol. 477, pp. 149-160, 2017.

[6] J. Tang, H. Jiang, Z. Li, M. Li, F. Liu, and Y. Wang, "A twolayer model for taxi customer searching behaviors using GPS trajectory data," IEEE Transactions on Intelligent Transportation Systems, vol. 17, no. 11, pp. 3318-3324, 2016.
[7] S. Zhang, J. Tang, H. Wang, Y. Wang, and S. An, "Revealing intra-urban travel patterns and service ranges from taxi trajectories," Journal of Transport Geography, vol. 61, pp. 72-86, 2017.

[8] Y. Zou, J. Tang, L. Wu, K. Henrickson, and Y. Wang, "Quantile analysis of factors influencing the time taken to clear road traffic incidents," Proceedings of the Institution of Civil Engineers Transport, vol. 170, no. 5, pp. 296-304, 2017.

[9] Y. Zou, K. Henrickson, D. Lord, Y. Wang, and K. Xu, "Application of finite mixture models for analysing freeway incident clearance time," Transportmetrica A: Transport Science, vol. 12, no. 2, pp. 99-115, 2016.

[10] J. Tang, G. Zhang, Y. Wang, H. Wang, and F. Liu, "A hybrid approach to integrate fuzzy C-means based imputation method with genetic algorithm for missing traffic volume data estimation," Transportation Research Part C: Emerging Technologies, vol. 51, pp. 29-40, 2015.

[11] J. Kwon and P. Varaiya, "Effectiveness of California's high occupancy vehicle (HOV) system," Transportation Research Part C: Emerging Technologies, vol. 16, no. 1, pp. 98-115, 2008.

[12] X. Liu, B. J. Schroeder, T. Thomson, Y. Wang, N. M. Rouphail, and Y. Yin, "Analysis of operational interactions between freeway managed lanes and parallel, general purpose lanes," Transportation Research Record, vol. 2262, pp. 62-73, 2011. 
[13] J. Tang, F. Liu, Y. Zou, W. Zhang, and Y. Wang, "An improved fuzzy neural network for traffic speed prediction considering periodic characteristic," IEEE Transactions on Intelligent Transportation Systems, pp. 1-11, 2017.

[14] Y. Zou, H. Yang, Y. Zhang, J. Tang, and W. Zhang, "Mixture modeling of freeway speed and headway data using multivariate skew-t distributions," Transportmetrica A: Transport Science, vol. 13, no. 7, pp. 657-678, 2017.

[15] L. Wu, Y. Zou, and D. Lord, "Comparison of sichel and negative binomial models in hot spot identification," Transportation Research Record, vol. 2460, no. 1, pp. 107-116, 2014.

[16] M. Burris and K. Lipnicky, "HOV or general purpose lanes?" Public Works Management and Policy, vol. 14, no. 2, pp. 130-147, 2009.

[17] B. Wright, Y. Zou, and Y. Wang, "Impact of traffic incidents on reliability of freeway travel times," Transportation Research Record, pp. 90-98, 2015.

[18] C. Carrion and D. Levinson, "Value of travel time reliability: a review of current evidence," Transportation Research Part A: Policy and Practice, vol. 46, no. 4, pp. 720-741, 2012.

[19] J. Tang, S. Zhang, X. Chen, F. Liu, and Y. Zou, “Taxi trips distribution modeling based on Entropy-Maximizing theory: A case study in Harbin city-China," Physica A: Statistical Mechanics and its Applications, vol. 493, pp. 430-443, 2018.

[20] Z. Li, D. A. Hensher, and J. M. Rose, "Willingness to pay for travel time reliability in passenger transport: a review and some new empirical evidence," Transportation Research Part E: Logistics and Transportation Review, vol. 46, no. 3, pp. 384-403, 2010.

[21] B. L. Smith, L. Qin, and R. Venkatanarayana, "Characterization of freeway capacity reduction resulting from traffic accidents," Journal of Transportation Engineering, vol. 129, no. 4, pp. 362368, 2003.

[22] V. L. Knoop, S. P. Hoogendoorn, and H. J. Van Zuylen, "Capacity reduction at incidents: Empirical data collected from a helicopter," Transportation Research Record, vol. 2071, pp. 19-25, 2008.

[23] H. Tu, H. Van Lint, and H. Van Zuylen, “The effects of traffic accidents on travel time reliability," in Proceedings of the 11th International IEEE Conference on Intelligent Transportation Systems (ITSC '08), pp. 79-84, December 2008.

[24] S. Turner, C. Naples, and R. Henk, "Travel time reliability of HOV facilities," in Proceedings of the 64th ITE Annual Meeting, Compendium of Technical Papers, 1994.

[25] W. Loudon, "Improving the estimation of potential travel-time savings from HOV lanes," in Proceedings of the Transportation Research Board Annual Meeting, Washington, DC, USA, 2007.

[26] X. Ye, K. Wang, Y. Zou, and D. Lord, "A semi-nonparametric Poisson regression model for analyzing motor vehicle crash data," PLoS ONE, vol. 13, no. 5, Article ID e0197338, pp. 1-17, 2018.

[27] T. Lomax and R. Margiotta, "Selecting travel reliability measures," 2003.

[28] H. Tu, H. Li, H. van Lint, and H. van Zuylen, "Modeling travel time reliability of freeways using risk assessment techniques," Transportation Research Part A: Policy and Practice, vol. 46, no. 10, pp. 1528-1540, 2012.

[29] H. Tu, J. W. C. Van Lint, and H. J. Van Zuylen, "Impact of traffic flow on travel time variability of freeway corridors," Transportation Research Record, vol. 1993, pp. 59-66, 2007.
[30] C. Chen, A. Skabardonis, and P. Varaiya, "Travel-time reliability as a measure of service," Transportation Research Record, vol. 1855, pp. 74-79, 2003.

[31] O. Franzese, L. Zhang, and A. Mahmoud, "Real-Time traffic information for emergency evacuations," Journal of Transportation Safety \& Security, vol. 2, no. 1, pp. 69-83, 2010.

[32] J. Tang, F. Liu, W. Zhang, S. Zhang, and Y. Wang, "Exploring dynamic property of traffic flow time series in multi-states based on complex networks: Phase space reconstruction versus visibility graph," Physica A: Statistical Mechanics and its Applications, vol. 450, pp. 635-648, 2016. 


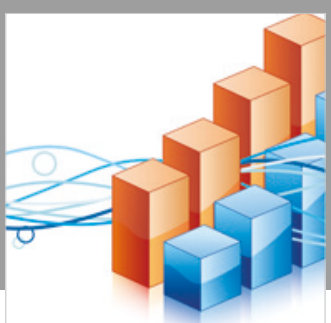

Advances in

Operations Research

\section{-n-m}
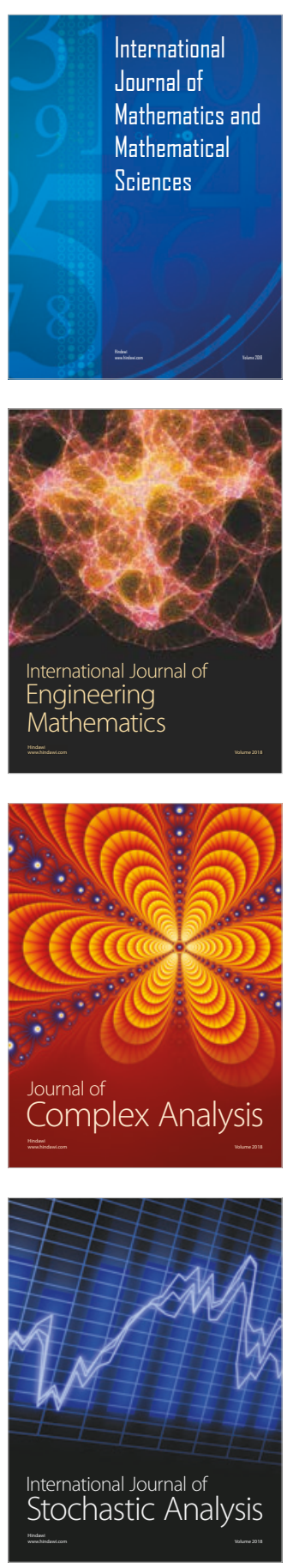
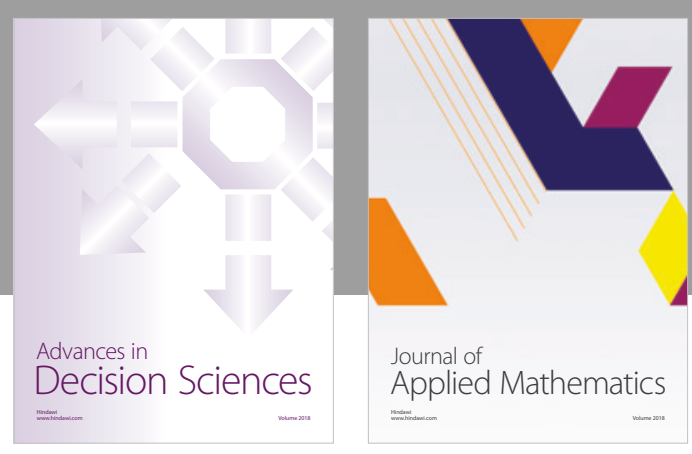

Journal of

Applied Mathematics
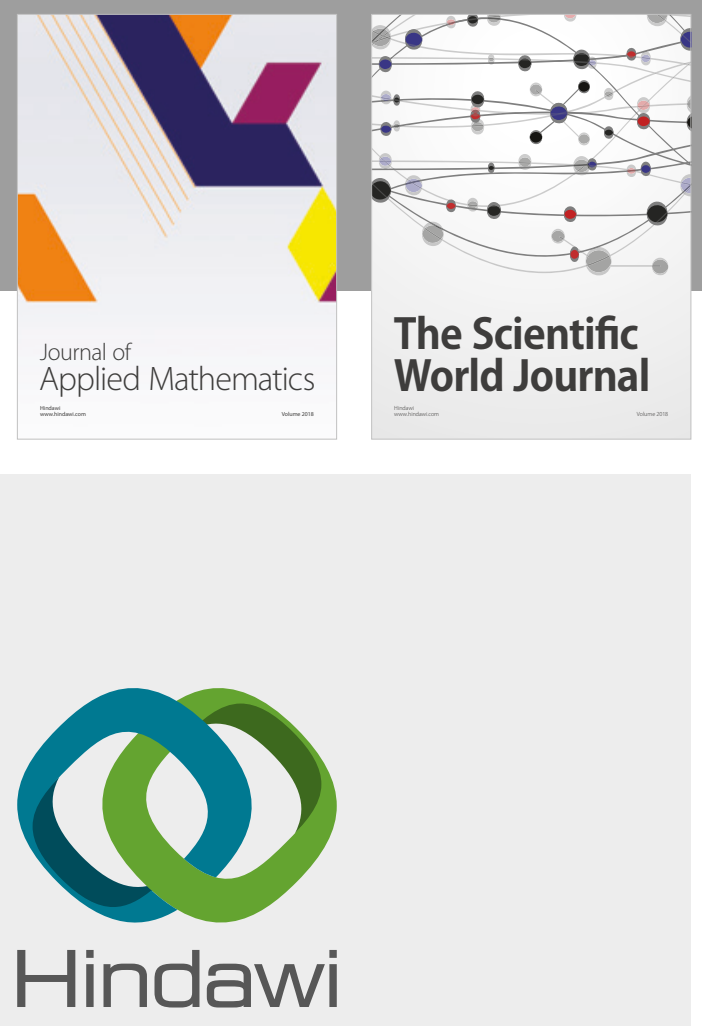

Submit your manuscripts at

www.hindawi.com

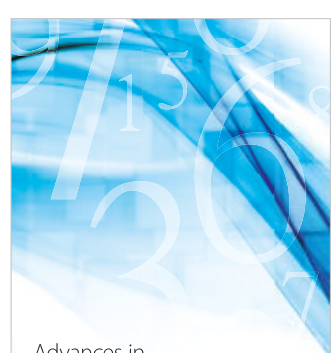

Advances in
Numerical Analysis
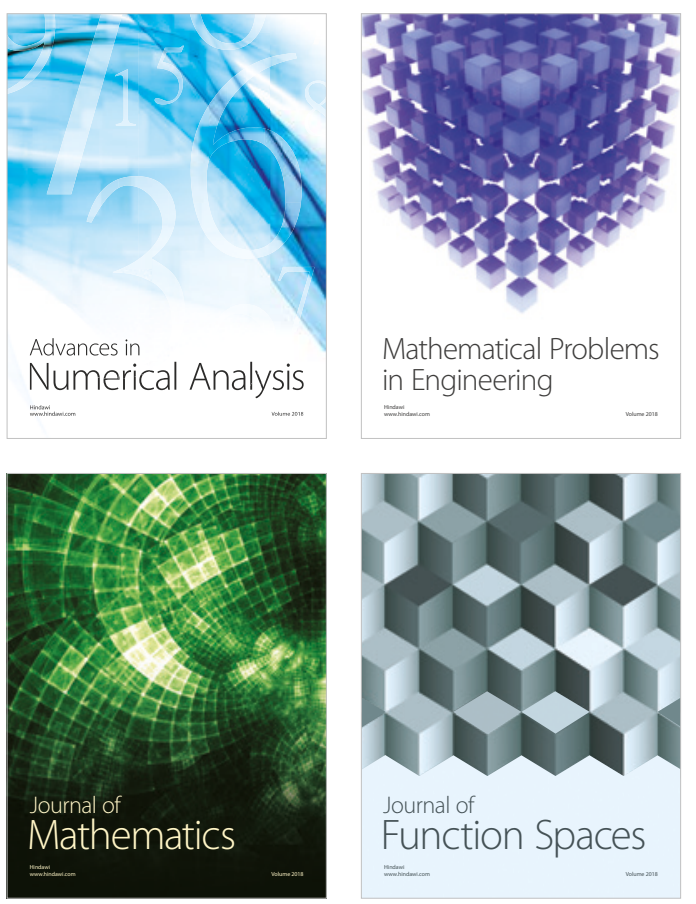

Mathematical Problems in Engineering

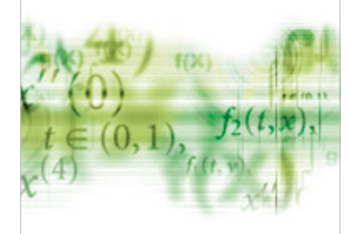

International Journal of

Differential Equations

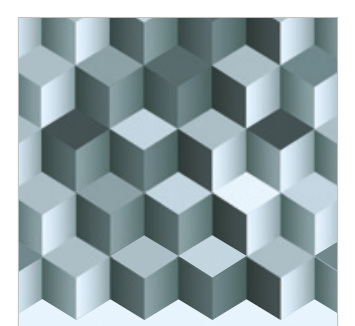

Journal of

Function Spaces

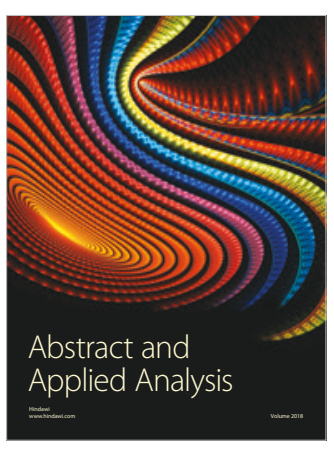

The Scientific

World Journal

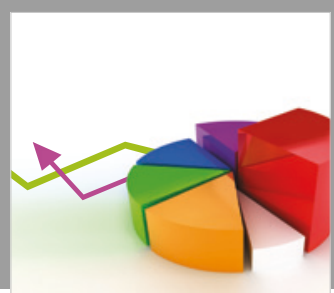

Journal of

Probability and Statistics
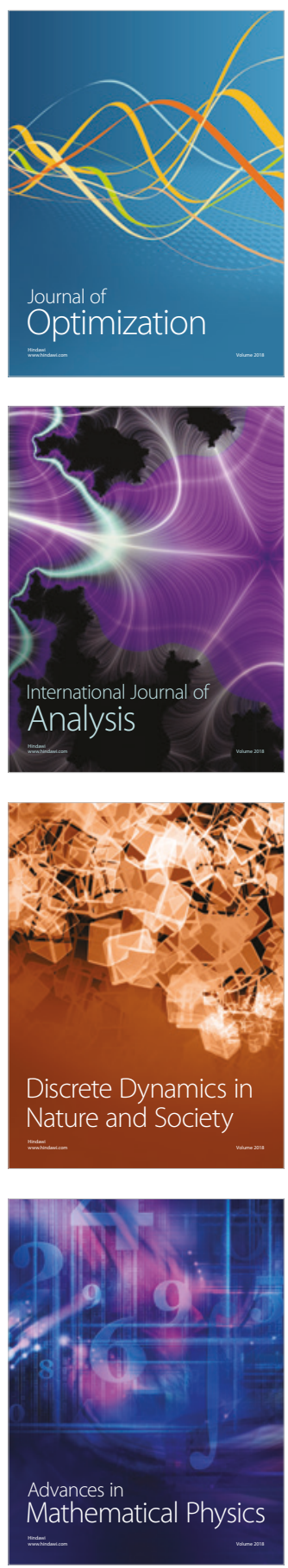